\title{
Artificial Intelligence to Improve Polyp Detection and Screening Time in Colon Capsule Endoscopy
}

Pere Gilabert ( $\nabla$ pere.gilabert@ub.edu )

Universitat de Barcelona

Jordi Vitrià

Universitat de Barcelona

Pablo Laiz

Universitat de Barcelona

Carolina Malagelada

University Hospital Vall d'Hebron

Angus Watson

Raigmore Hospital, NHS Highland

Hagen Wenzek

CorporateHealth International ApS

Santi Seguí

Universitat de Barcelona

\section{Research Article}

Keywords:

Posted Date: January 31st, 2022

DOI: https://doi.org/10.21203/rs.3.rs-1278962/v1

License: (c) (1) This work is licensed under a Creative Commons Attribution 4.0 International License.

Read Full License 


\title{
Artificial Intelligence to improve polyp detection and screening time in colon capsule endoscopy
}

\author{
Pere Gilabert $^{1, *}$, Jordi Vitrià ${ }^{1}$, Pablo Laiz ${ }^{1}$, Carolina Malagelada ${ }^{2,3}$, Angus Watson $^{4}$, \\ Hagen Wenzek ${ }^{5}$, and Santi Seguí ${ }^{1}$
}

\author{
${ }^{1}$ Departament de Matemàtiques i Enginyeria Informàtica, Universitat de Barcelona, Barcelona, Spain \\ ${ }^{2}$ Digestive System Research Unit, University Hospital Vall d'Hebron, Barcelona, Spain \\ ${ }^{3}$ Department of Medicine, Universitat Autònoma de Barcelona, Barcelona, Spain \\ ${ }^{4}$ Department of Colorectal Surgery, Raigmore Hospital, NHS Highland, Inverness, Scotland \\ ${ }^{5}$ CorporateHealth International ApS \\ *pere.gilabert@ub.edu
}

\begin{abstract}
Colon Capsule Endoscopy (CCE) is a minimally invasive procedure which is increasingly being used as an alternative to conventional colonoscopy. Videos recorded by the capsule cameras are long and require one or more experts' time to review and identify polyps or other potential intestinal problems that can lead to major health issues. We developed and tested a multi-platform web application, Al-Tool, which embeds a Convolution Neural Network (CNN) to help CCE reviewers. With the help of artificial intelligence, Al-Tool is able to detect images with high probability of containing a polyp and prioritize them during the reviewing process. With the collaboration of 3 experts that reviewed 18 videos, we compared the classical linear review method using RAPID Reader Software v9 and the new software we present. Applying the new strategy, reviewing time was reduced by a factor of 6 and polyp detection sensitivity was increased from $81.08 \%$ to $87.80 \%$.
\end{abstract}

\section{Introduction}

Colorectal cancer is the third most common type of cancer worldwide and ranks second on the list of most aggressive and deadly cancers ${ }^{1}$. According to the Global Cancer Observatory, out of an estimated total of 1.9 million cases in 2020 , this disease has caused the death of more than 935,000 people worldwide ${ }^{1}$. This type of cancer has also been linked to unhealthy lifestyle habits such as smoking, alcohol consumption, unhealthy diets or sedentary lifestyle $\mathrm{e}^{2,3}$. A clear relationship has been drawn between colon cancer and obesity ${ }^{4,5}$ along with genetic predisposition ${ }^{6}$.

One of the initial signs of the development of colon cancer is the appearance of polyps in the colon that grow in an uncontrolled manner ${ }^{7}$. The detection of polyps when they are still small is crucial to prevent their transformation into cancer. Screening programs are aimed to detect early-stage cancer, improving the patient's chances of survival ${ }^{8,9}$. With the disruption of colonoscopy screening programs due to the COVID-19 pandemic, an increase in incidence of $0.2-0.9 \%$ and deaths of $0.6-1.6 \%$ is predicted over the next 30 years $(2020-2050) .{ }^{10}$. To be able to mitigate these effects, well-resourced screening programs are urgently needed. Unfortunately, not all healthcare systems can afford a large increase in demand for colonoscopy which is usually a primary or secondary screening test. Therefore, other equally effective methods should be considered ${ }^{10,11}$. Among those alternatives, CCE has proven to be one of the most safe and effective tools, with an accuracy very similar to that of traditional colonoscopies ${ }^{12}$. It is less invasive for the patient ${ }^{13}$, it does not cause discomfort during the procedure (only minimal discomfort before the procedure due to required bowel preparation ${ }^{13}$ ) and no anesthesia is needed. However, the reading of CCE is time-consuming and requires qualified medical personnel ${ }^{14-16}$. For CCE to be considered as an alternative procedure in colorectal cancer detection, the use of AI could streamline the process without compromising accuracy ${ }^{17-19}$.

AI has been extensively applied to medical imaging problems ${ }^{20}$. In the colon capsule endoscopy field, multiple methods have been presented to automatically detect ulcers ${ }^{21}$, polyps ${ }^{22,23}$, Crohn's disease ${ }^{24}$ or bowel cleanliness $^{25}$. These methods have shown promising results, but they are generally validated with limited data or biased datasets which does not guarantee a good generalization in clinical practice ${ }^{18,26}$. There is no doubt that AI has potential benefits to both doctors and patients, but its application to the clinical practice is challenging. AI is not yet at a point where it can completely replace the intervention by human experts ${ }^{18}$. Although U.S. Food and Drug Administration (FDA) has approved some assistance algorithms ${ }^{27}$, no guidelines establishing the role of AI currently exist. In order to achieve this, these systems would need to gain confidence of medical experts and ethical and regulatory issues would need to be solved ${ }^{15,28}$. This is why it is important to develop systems 
that cooperate with the experts, facilitating their decision making.

In this paper, we present a novel CNN-based system, AI-Tool, to assist physicians with the detection of colonic polyps. Given a video, it outputs a probability score per image frame to contain a polyp and a heatmap explaining the reasoning of the prediction. This heatmap, allows the expert to focus on the area of the image where the CNN suggests the polyp to be located. An experiment performed with 18 videos revised by 3 expert readers, has shown that the proposed system reduces screening time significantly while it also increases the sensitivity of polyp detection.

\section{Methods}

\section{Study Population}

Eighteen videos of patients with polyps obtained using the PillCam COLON 2 capsule (Medtronic) were randomly selected for this experiment. Bowel preparation in accordance with a standardized cleansing protocol was performed in all patients. All videos were obtained using PillCam COLON 2 which has two heads (front and rear). They were anonymized to protect patient information. Patients' mean age was $58.1 \pm 18.7$ years (range, 18-92 years) and mean colon transit time was $4 \mathrm{~h} 10$ min (range, $0.17-14.2$ hours).

Before the experiments began, the two videos obtained from each of the heads of the capsule from each patient's video were meticulously reviewed by four independent CCE readers in order to create the ground truth for the experiments (gold standard). Each detected polyp was assigned a unique identifier, the timestamp of the first and the last image where the polyp was visible, and from which head it was reported. The independent analysis of the experts was then shared with all experts, reaching a consensus in case of discrepancies. During this process, a total of 52 unique polyps were found. The video with the most polyps had 7 , while there were 5 videos with only one polyp.

The polyps' size was estimated with RAPID. A total of 23 polyps were identified as large $(\geq 6 \mathrm{~mm})$ and 29 polyps as small $(<6 \mathrm{~mm})$. There were 5 polyps larger than $10 \mathrm{~mm}$ and only one polyp smaller than $3 \mathrm{~mm}$.

\section{Experimental Design}

Three experienced CCE readers reviewed the videos selected for this experiment. Each reader reviewed half of the videos using the standard RAPID Reader Software v9 (Medtronic) and the other half using the AI-Tool. Results obtained by the experts using each of the tools are reported in terms of number of polyps detected (sensitivity) as well as time needed to complete the reviews (screening time).

The experiment conducted in this study was restricted to the images of the colon. Identification of the entrance and exit of the colon was previously provided to the readers. When they used RAPID software, they were asked to perform the standard screening procedure without any screening time limitation. For the AI-Tool, the review time was limited to 30 minutes regardless of the video's length.

For both tools, readers were required to review the videos without pauses or external stimuli that could lead to distractions. During the review, the readers labelled the images that they identified as a polyp using the tools provided within each of the applications. In the case of RAPID, experts were asked to tag all the unique polyps they found. When using the AI-Tool, experts were asked to make a decision, polyp, clear or other, for each sequence that was presented to them.

\section{CCE Readers}

The expert readers are endoscopy nurses with at least 2 years of experience with CCE. They have a formal CCE training and conduct between 5-20 video analyses per week. On a daily basis, they follow a standard operating procedure to ensure that each video is analyzed in a consistent, repeatable way and documented according to common standards.

\section{Screening Tool: RAPID Reader v9.0}

RAPID Reader v9.0 is a Medtronic proprietary software, used to review and interpret wireless capsule endoscopy videos. This software is widely used by colon capsule endoscopy professionals designed to review the video in a comfortable way. The video is shown in temporal order and the app allows to label images, to add comments to images and to measure any part of the image with an integrated ruler function. The user can change the replay speed of the video at any time and the application shows the approximate area of the abdomen where the capsule is located. It also warns the user if the capsule is passing through an area at high speed, so the user can know if special attention is required. RAPID has several modes to review videos: single view with the frontal camera, twin head mode showing both cameras simultaneously (Figure 1) and collage mode which shows a batch of frames selected by traditional computer vision techniques.

\section{Screening Tool: Al-Tool}

The AI-Tool is a software designed to assist clinicians in the detection of polyps, by complementing any proprietary video reviewing software, such as RAPID. It embeds a Convolutional Neural Network (CNN) into a web tool that presents images 


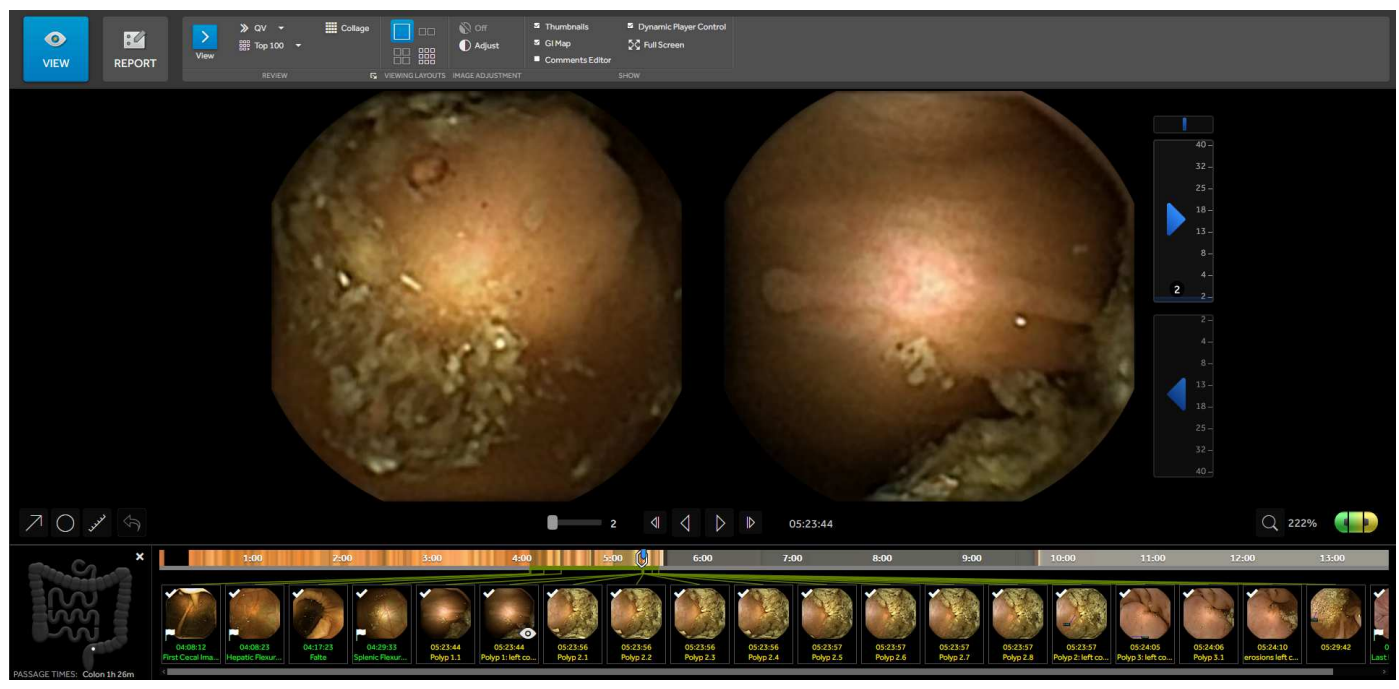

Figure 1. RAPID software: screen with images from both camera heads (green/yellow) and marked thumbnails.

with potential polyps to the user in a sequence of declining certainty. Therefore, images that are very likely to contain a polyp will appear first. The embedded CNN has been trained with 120 CCE videos (2,080 polyp images and 246k negative images), using a deep metric learning strategy ${ }^{29}$ in order to deal with a rather small and imbalanced dataset. The experimental validation of the network has shown a sensitivity over $90 \%$ at a specificity of $95 \%$ when evaluated in a fully automatic setting (when no expert is involved) using full videos. All 120 videos used in the training of the CNN were excluded for this experiment.

The AI-Tool computes two outputs using a CNN: a probability score per frame to contain a polyp and a heatmap to visualize the reasoning behind the score. The AI-Tool is then able to select and show those candidate images to the reader sorted by probability.

Each potential polyp image is displayed along with eight context frames, the four preceding and the four following it (Figure 2). For each frame, a colored square is shown to indicate the probability of it being a polyp using a colorblind friendly palette that can be customized when the application starts. Each image can be enlarged by clicking on it, then further information is presented such as the probability or the timestamp of the sequence. The image sequence can be also displayed as a video using the left and right keyboard arrows. Heatmaps can always be activated (Figure 3, row 2) showing the most likely area to contain a polyp. A further benefit of the heatmap is that it helps readers to understand the reasoning behind the polyp probability and as a consequence, increase their trust in the system.

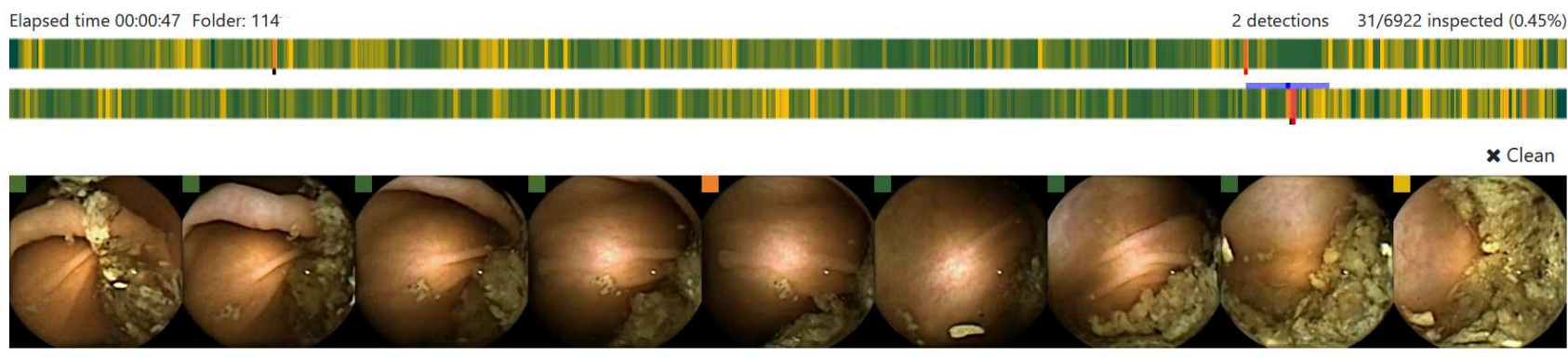

Figure 2. Candidate polyp sequence displayed in the AI-Tool. Each colored bar shows the probabilities for a polyp in one head of the capsule. The proposed image is presented in the center frame and 4 context images are placed by each side.

\section{Human rights statement and informed consent}

The study was conducted in accordance with the guidelines of the Declaration of Helsinki and was approved by the University of Barcelona's Bioethics Commission, Institutional Review Board IRB00003099. The patients were informed of the risk and benefits of $\mathrm{CCE}$ at the time of the procedure, and all provided written informed consent to use their de-identified data for research purposes. 


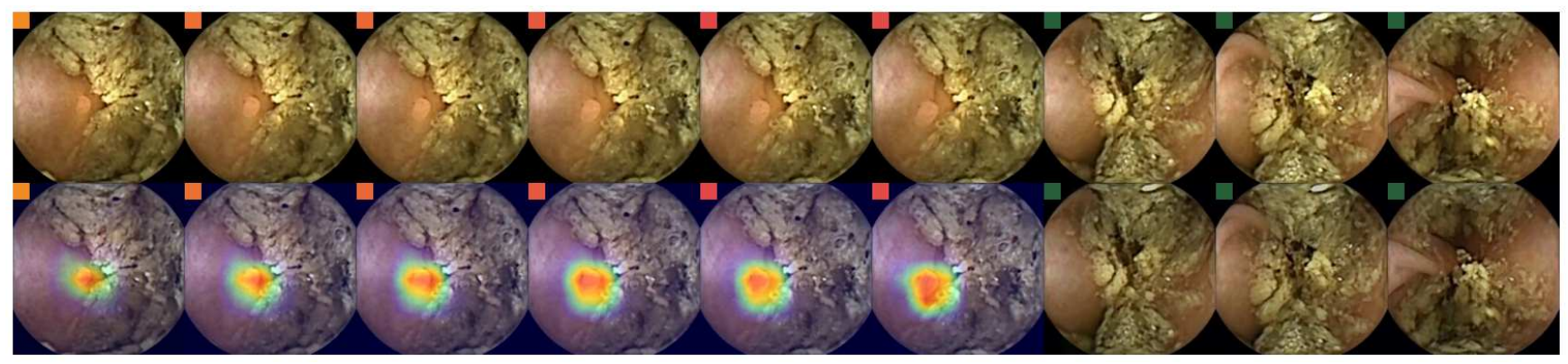

Figure 3. A sequence of images (top). Same sequence with heatmaps activated (bottom). Each frame's score is shown in the top-left corner. The score is encoded in a green-red scale where red stands for higher polyp probability. The higher this score, the higher the heatmap intensity.

\section{Results}

\section{Polyp detection}

The overall sensitivity of polyp detection using RAPID as the screening procedure was $81.08 \%$ while using the AI-Tool the sensitivity increased to $87.80 \%$. Table 1 shows the percentage of polyps found using both tools distinguishing between two categories: polyp size (in millimeters) and visibility (in number of frames). The sensitivity using RAPID turned out to be $76.92 \%$ for polyps smaller than $6 \mathrm{~mm}$ and $85.71 \%$ for larger polyps. Both numbers increased when the AI-Tool was used ( $85.42 \%$ and $91.18 \%$ for small and large polyps respectively).

The biggest difference between both tools was observed in the visibility of the polyp. For polyps appearing in a few frames (low visibility), the table shows a significant improvement when using the AI-Tool. While RAPID achieved an accuracy of $58.33 \%$, the AI-Tool reached $80.00 \%$. This represents an increase of $21.67 \%$ in this category. Smaller improvements using the AI-Tool were also reported for polyps appearing in a larger number of frames.

These results show that small polyps and polyps that appear for only a few frames are more likely to be detected using the AI-Tool than using the RAPID application.

Table 1. Detection of polyps distinguishing by size and visibility.

\begin{tabular}{c|c|cc}
\hline & \#Polyps & RAPID & AI-Tool \\
\hline Polyps & 52 & $81.08 \%$ & $87.80 \%$ \\
\hline Small Polyps $(<6 \mathrm{~mm})$ & 29 & $76.92 \%$ & $85.42 \%$ \\
Large Polyps $(\geq 6 \mathrm{~mm})$ & 23 & $85.71 \%$ & $91.18 \%$ \\
\hline Low visibility $(\leq 3$ frames $)$ & 9 & $58.33 \%$ & $80.00 \%$ \\
Normal visibility $(4-10$ frames $)$ & 15 & $73.91 \%$ & $81.82 \%$ \\
High visibility $(>10$ frames $)$ & 28 & $92.31 \%$ & $93.33 \%$ \\
\hline
\end{tabular}

\section{CCE Screening Time}

One of the aims of this study was to compare the time needed for the detection of polyps using RAPID and our AI-Tool. The average time required for the experiments performed with RAPID was 47.11 minutes (11.6 minutes for each hour of CCE video reviewed) with a maximum of 126 minutes. Let us recall that the time for analysis using the AI-Tool was fixed at 30 minutes for all the experiments.

Figure 4 shows the average sensitivity curve as a function of time for both applications. AI-Tool took only 8.00 minutes to reach the same accuracy as RAPID (point A). Since the mean of RAPID experiments was 47.11 minutes we can state that the AI-Tool reduces the time needed to reach the same accuracy as RAPID by a factor of $47.11 / 8.00 \approx 6$. We can also see that RAPID experiments reached a $55.41 \%$ of sensitivity (point B) by minute 30 when the AI-Tool experiments finished.

The shape of the curves is also an aspect worth considering. While the RAPID curve has an almost linear behavior, the AI-Tool curve shows an initial steep slope. In the first 10 minutes of the analysis $84.14 \%$ of the polyps are detected. In the next 10 minutes, this number rises to $86.59 \%$, which represents an increase of $2.45 \%$ in this period. Finally, only $1.21 \%$ of the polyps are detected in the last 10 minutes of the experiment. This indicates that our application is proposing the relevant images in the first minutes of visualization. 


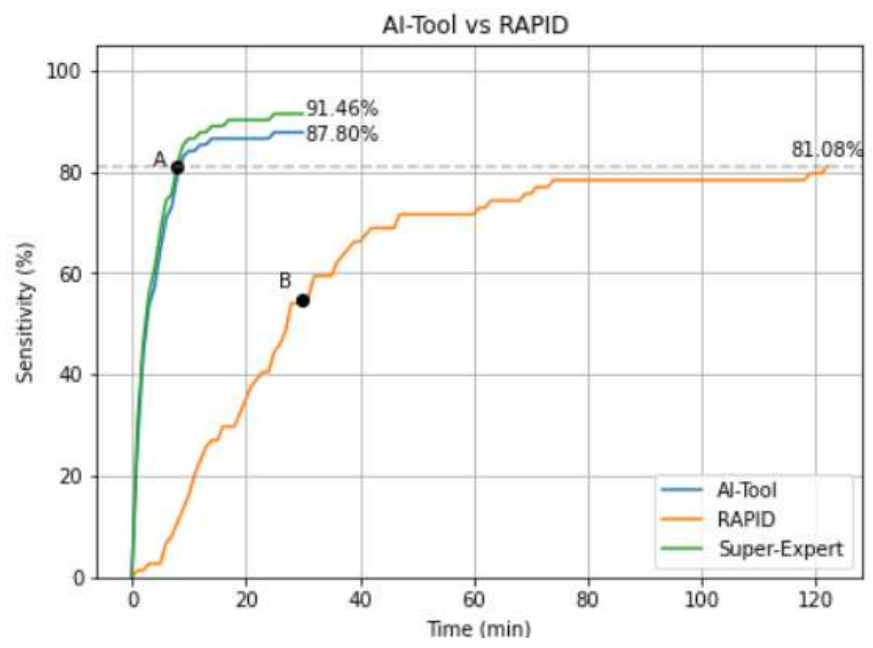

Figure 4. Mean sensitivity curve of the experiments using both applications. In green the Super-Expert curve (gold standard) that represents the maximum value that the blue line could reach. This curve has been calculated simulating an expert who never makes mistakes when identifying a polyp while using the AI-Tool.

\section{Qualitative Results}

The following are some qualitative results obtained during the experiment. We focus on showing the images of polyps that have not been detected with either of the two applications (specificity).

Figure 5 shows those polyps not detected with the AI-Tool because of the imposed time restriction ( 30 minutes). The score given to these image does not exceed 15\%, therefore, the experts never reviewed them. In fact, these four images are the polyps with the lowest score of this study. The polyps of these images are difficult to find since they are partially occluded or do not present a regular morphology.
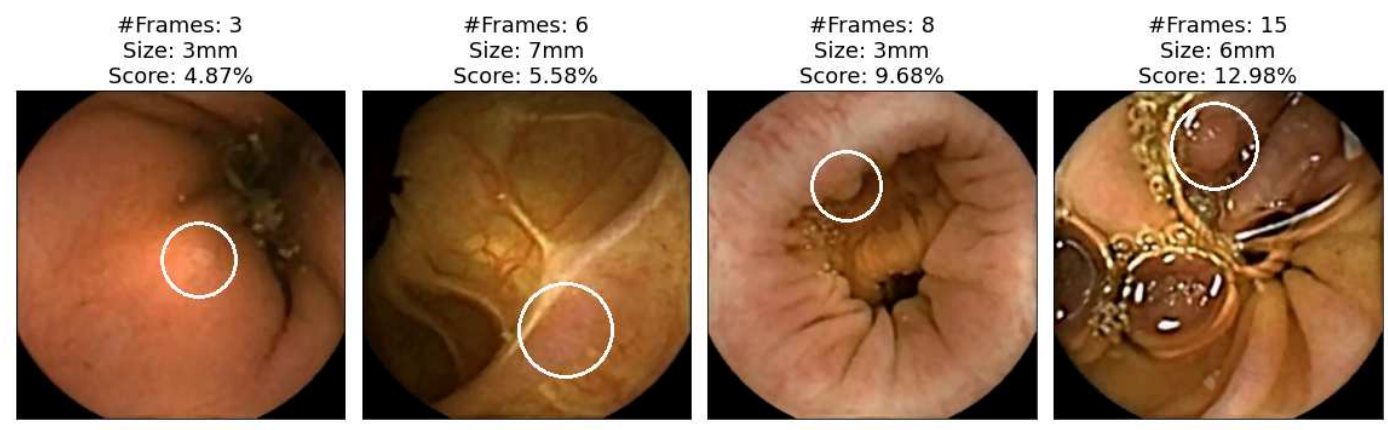

Figure 5. Polyp frames to which the app has attributed a small score and, therefore, none of the experts have been able to review in the first 30 minutes. Polyps are circled in white.

Figure 6 shows images of polyps reviewed and discarded by all the experts even though the AI-Tool assigned them a remarkably high probability. These missed polyps are the result of human error or discrepancies between the video reviewer and the experts who generated the ground truth.

Finally, Figure 7 shows some examples of polyp images missed in RAPID experiments but correctly detected using the AI-Tool. Due to the size of the polyp and the fast movement of the capsule that took few images, these polyps are especially difficult to find using RAPID. In contrast, they are easy to find for AI-Tool users as they are presented with the clearest image.

\section{Conclusions}

In this paper, thanks to the AI enhancement, we mitigate one of the main drawbacks of CCE, the required time for the analysis while increasing the detection rate of the experts. We consider that these improvements further improve the value proposition of $\mathrm{CCE}$ as a clinically viable and cost-effective alternative to traditional imaging methods of the gastrointestinal tract.

The proposed method, AI-Tool, uses the output of a CNN architecture. It scores frames based on the probability of 

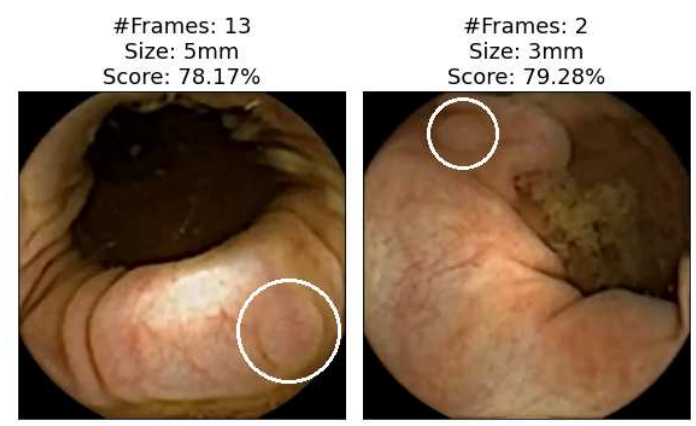

Figure 6. Images that all experts have reviewed and found not to be polyps. Polyps are circled in white.
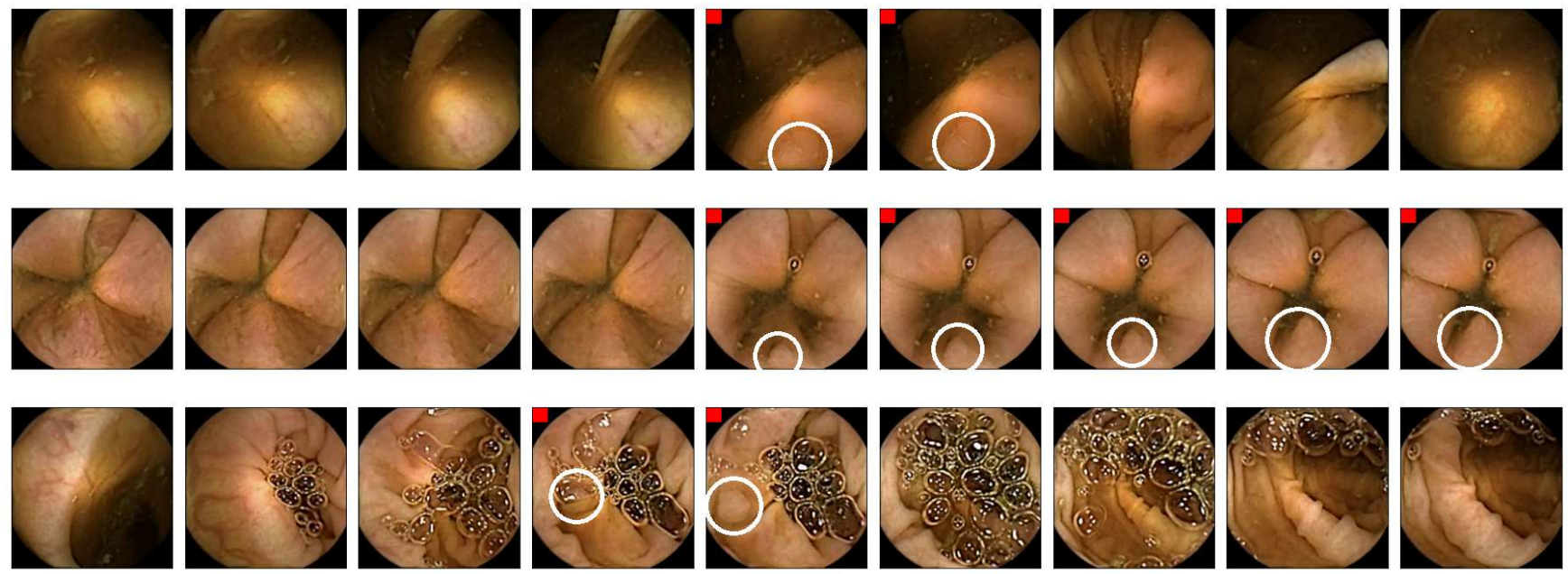

Figure 7. Example of polyps missed in Rapid experiments. Polyp frames are tagged with a red square. Polyps are circled in white. 
containing a polyp and then it reorders the video images to present the most relevant ones first. The validation was performed by three clinical experts that analyzed 18 videos, comparing the standard method with and without the proposed AI-enhanced application. With the assistance of the AI-Tool, the time required to review the videos was reduced by a factor of 6 and the sensitivity increased from $81.08 \%$ to $87.80 \%$. In the case of small polyps $(<6 \mathrm{~mm})$, the improvement in sensitivity obtained by the AI-Tool was $8.50 \%$. and for polyps with low visibility (seen in $\leq 3$ frames), the improvement in detection was $21.67 \%$.

\section{References}

1. Sung, H. et al. Global cancer statistics 2020: Globocan estimates of incidence and mortality worldwide for 36 cancers in 185 countries. CA: A Cancer J. for Clin. 71, 209-249, DOI: https://doi.org/10.3322/caac.21660 (2021). https: //acsjournals.onlinelibrary.wiley.com/doi/pdf/10.3322/caac.21660.

2. O'Sullivan, D. E. et al. Combinations of modifiable lifestyle behaviours in relation to colorectal cancer risk in alberta's tomorrow project. Sci. reports 10, 1-11 (2020).

3. Ahmed, M. Colon cancer: a clinician's perspective in 2019. Gastroenterol. research 13, 1 (2020).

4. Ye, P., Xi, Y., Huang, Z. \& Xu, P. Linking obesity with colorectal cancer: Epidemiology and mechanistic insights. Cancers 12, DOI: 10.3390/cancers12061408 (2020).

5. Lauby-Secretan, B. et al. Body fatness and cancer-viewpoint of the iarc working group. New Engl. J. Medicine 375, 794-798 (2016).

6. Valle, L. Genetic predisposition to colorectal cancer: where we stand and future perspectives. World journal gastroenterology 20, 9828-9849, DOI: 10.3748/wjg.v20.i29.9828 (2014). 25110415[pmid].

7. Bond, J. Colon polyps and cancer. Endoscopy 35, 27-35 (2003).

8. Levin, T. R. et al. Effects of organized colorectal cancer screening on cancer incidence and mortality in a large communitybased population. Gastroenterology 155, 1383-1391 (2018).

9. Loveday, C. et al. Prioritisation by fit to mitigate the impact of delays in the 2-week wait colorectal cancer referral pathway during the covid-19 pandemic: a uk modelling study. Gut 70, 1053-1060 (2021).

10. de Jonge, L. et al. Impact of the covid-19 pandemic on faecal immunochemical test-based colorectal cancer screening programmes in australia, canada, and the netherlands: a comparative modelling study. The Lancet Gastroenterol. \& Hepatol. 6, 304-314 (2021).

11. Laghi, L., Cameletti, M., Ferrari, C. \& Ricciardiello, L. Impairment of colorectal cancer screening during the covid-19 pandemic. The Lancet Gastroenterol. \& Hepatol. 6, 425-426 (2021).

12. Vuik, F. E. et al. Colon capsule endoscopy in colorectal cancer screening: a systematic review. Endoscopy (2021).

13. Ismail, S., Murphy, G., Semenov, S. \& McNamara, D. Comparing colon capsule endoscopy to colonoscopy; a symptomatic patient's perspective, DOI: 10.21203/rs.3.rs-362903/v1 (2021).

14. Maieron, A. et al. Multicenter retrospective evaluation of capsule endoscopy in clinical routine. Endoscopy 36, 864-868 (2004).

15. Koulaouzidis, A., Dabos, K., Philipper, M., Toth, E. \& Keuchel, M. How should we do colon capsule endoscopy reading: a practical guide. Ther. Adv. Gastrointest. Endosc. 14, 26317745211001983 (2021).

16. Rondonotti, E., Pennazio, M., Toth, E. \& Koulaouzidis, A. How to read small bowel capsule endoscopy: a practical guide for everyday use. Endosc. Int. Open 8, E1220-E1224 (2020).

17. Byrne, M. F. \& Donnellan, F. Artificial intelligence and capsule endoscopy: Is the truly "smart" capsule nearly here? Gastrointest. endoscopy 89, 195-197 (2019).

18. Yang, Y. J. The future of capsule endoscopy: The role of artificial intelligence and other technical advancements. Clin. Endosc. 53, 387 (2020).

19. Chetcuti Zammit, S. \& Sidhu, R. Capsule endoscopy-recent developments and future directions. Expert. Rev. Gastroenterol. \& Hepatol. 15, 127-137 (2021).

20. Topol, E. J. High-performance medicine: the convergence of human and artificial intelligence. Nat. Medicine 25, 44-56, DOI: 10.1038/s41591-018-0300-7 (2019).

21. Rahim, T., Usman, M. A. \& Shin, S. Y. A survey on contemporary computer-aided tumor, polyp, and ulcer detection methods in wireless capsule endoscopy imaging. Comput. Med. Imaging Graph. 101767 (2020). 
22. Laiz, P. et al. Wce polyp detection with triplet based embeddings. Comput. Med. Imaging Graph. 86, 101794, DOI: https://doi.org/10.1016/j.compmedimag.2020.101794 (2020).

23. Jin, E. H. et al. Improved accuracy in optical diagnosis of colorectal polyps using convolutional neural networks with visual explanations. Gastroenterology 158, 2169-2179.e8, DOI: https://doi.org/10.1053/j.gastro.2020.02.036 (2020).

24. Goran, L., Negreanu, A. M., Stemate, A. \& Negreanu, L. Capsule endoscopy: Current status and role in crohn's disease. World journal gastrointestinal endoscopy 10, 184 (2018).

25. Noorda, R., Nevárez, A., Colomer, A., Beltrán, V. P. \& Naranjo, V. Automatic evaluation of degree of cleanliness in capsule endoscopy based on a novel cnn architecture. Sci. Reports 10, 1-13 (2020).

26. Saurin, J.-C., Beneche, N., Chambon, C. \& Pioche, M. Challenges and future of wireless capsule endoscopy. Clin. endoscopy 49, 26 (2016).

27. Muehlematter, U. J., Daniore, P. \& Vokinger, K. N. Approval of artificial intelligence and machine learning-based medical devices in the usa and europe (2015-20): a comparative analysis. The Lancet Digit. Heal. (2021).

28. Ahmad, O. F., Stoyanov, D. \& Lovat, L. B. Barriers and pitfalls for artificial intelligence in gastroenterology: ethical and regulatory issues. Tech. Innov. Gastrointest. Endosc. 22, 80-84 (2020).

29. Laiz, P. et al. Wce polyp detection with triplet based embeddings. Comput. Med. Imaging Graph. 86, 101794 (2020).

\section{Acknowledgements}

The authors would like to thank the team from CorporateHealth International ApS for their feedback. This work has been also supported by MINECO Grant RTI2018-095232-B-C21, SGR 1742, Instituto de Salud Carlos III as well as the Innovate UK project 104633 .

\section{Author contributions}

All authors contributed to the study conception and design. Data collection was performed by H. W. The first draft of the manuscript was written by P. G. and all authors commented on previous versions of the manuscript. All authors read and approved the final manuscript.

\section{Competing interests}

H.W. is co-founder of CorporateHealth International, a company that may be affected by the research reported in the enclosed paper. H.W. has not been involved in the process of analyzing the results and had no prior knowledge of whether or not the videos provided contained polyps. No potential competing interest was reported by the other authors.

\section{Data availability}

The data used during the present study are not publicly available as they are property of National Services Scotland (NHS Highland) but are available through co-author A.W. (angus.watson@nhs.scot) upon reasonable request. 